\title{
Global Exponential Stability of Periodic Solutions for a Nicholson's Blowflies Model with a Linear Harvesting Term
}

\author{
Ani Jiang* \\ College of Mathematics and Computer Science, Hunan University of Arts and Science, Changde, Hunan 415000, P.R. China
}

Received: 5 Oct. 2013, Revised: 2 Jan. 2014, Accepted: 3 Jan. 2014

Published online: 1 Sep. 2014

\begin{abstract}
In this paper, we study a generalized Nicholson's blowflies model with a linear harvesting term, which is defined on the positive function space. Under proper conditions, we employ a novel proof to establish some criteria for the existence and global exponential stability of positive periodic solutions for this model. Moreover, we give an example and its numerical simulations to illustrate our main results.
\end{abstract}

Keywords: Nicholson's blowflies model; linear harvesting term; positive periodic solution; global exponential stability.

\section{Introduction}

It is well known that the Nicholson's blowflies equation has been introduced by Gurney et al. [1] to describe the population of the Australian sheep-blowfly and to agree with the experimental data obtained in [2]. Later, the theory of Nicholson's blowflies equation has been made a remarkable progress in the past many years. Recently, L. Berezansky et al. [3] had made a review about the Nicholsons blowflies differential equations, and presented the following model proposed the following Nicholson's blowflies model:

$$
\begin{gathered}
x^{\prime}(t)=-\delta x(t)+p x(t-\tau) e^{-a x(t-\tau)}-H x(t-\sigma), \\
\delta, p, \tau, a, H, \sigma \in(0,+\infty),
\end{gathered}
$$

where $H x(t-\sigma)$ is a linear harvesting term, $x(t)$ is the size of the population at time $t, p$ is the maximum per capita daily egg production, $\frac{1}{a}$ is the size at which the population reproduces at its maximum rate, $\delta$ is the per capita daily adult death rate, and $\tau$ is the generation time.

In the real world phenomena, the variation of the environment plays an important role in many biological and ecological dynamical systems. In particular, the effects of a periodically varying environment are important for evolutionary theories, as the selective forces on systems in a fluctuating environment differ from those in a stable environment. Thus, the assumption of periodicity of the parameters of the system (in a way) incorporates the periodicity of the environment (e.g., seasonal effects of weather, food supplies, mating habits, etc.). A very basic and important ecological problem associated with study of multi species population interactions in a periodic environment is the existence of positive periodic solution which plays the role of the equilibrium in the autonomous models. Consequently, (1.1) has been naturally extended to the generalized equations with time-varying coefficient and delays. In particular, some criteria were established in $[4,5,6,7]$ to guarantee the existence of positive periodic solutions for (1.1) and its generalized equations by applying the method of coincidence degree and the fixed-point theorem in cones; several sufficient conditions were also obtained in $[9,10]$ to ensure that the solutions of its generalized system converge locally exponentially to a positive almost periodic solution. However, as pointed out by [11], it is difficult to study the global dynamic behaviors of the Nicholson's blowflies model with a linear harvesting term. So far, there is no literature considering the global exponential stability of positive periodic solutions for (1.1) and its generalized equations. Thus, it is also a unsolved open problem to reveal the global dynamic behaviors of positive periodic solutions for Nicholson's blowflies model with the linear harvesting term.

\footnotetext{
*Corresponding author e-mail: jiangani@aliyun.com
} 
Motivated by the above discussions, the main purpose of this paper is to establish some criteria for the global exponential stability of positive periodic solutions for a general Nicholson's blowflies model with the linear harvesting term given by

$$
\begin{aligned}
x^{\prime}(t)= & -a(t) x(t)+\sum_{j=2}^{m} \beta_{j}(t) x\left(t-\tau_{j}(t)\right) e^{-\gamma_{j}(t) x\left(t-\tau_{j}(t)\right)} \\
& +\beta_{1}(t) x\left(t-\tau_{1}(t)\right) e^{-\gamma_{1}(t) x(t)}-H(t) x(t-\sigma(t)),(1.2)
\end{aligned}
$$

where $a, H \sigma, \gamma_{j}: \mathbb{R} \rightarrow(0,+\infty)$ and $\beta_{j}, \tau_{j}: \mathbb{R} \rightarrow[0,+\infty)$ are continuous $T$-periodic functions for $j=1,2, \ldots, m$ with $T>0$. Obviously, (1.1) is a special case of (1.2) with constant coefficients and delays.

For convenience, we introduce some notations. In the following part of this paper, given a bounded continuous function $g$ defined on $R$, let $g^{+}$and $g^{-}$be defined as

$$
g^{+}=\sup _{t \in \mathbb{R}} g(t), g^{-}=\inf _{t \in \mathbb{R}} g(t) .
$$

It will be assumed that

$$
\gamma_{j}^{-} \geq 1(j=1,2, \ldots, m), r:=\max \left\{\max _{1 \leq j \leq m} \tau_{j}^{+}, \sigma^{+}\right\} .
$$

Throughout this paper, let $C=C([-r, 0], \mathbb{R})$ be the continuous functions space equipped with the usual supremun norm $\|\cdot\|$, and let $C_{+}=C([-r, 0],(0,+\infty))$. If $x$ is continuous and defined on $\left[-r+t_{0}, \sigma\right)$ with $t_{0}, \sigma \in \mathbb{R}$, then we define $x_{t} \in C$ where $x_{t}(\theta)=x(t+\theta)$ for all $\theta \in[-r, 0]$.

Due to the biological interpretation of model (1.2), only positive solutions are meaningful and therefore admissible. Thus we just consider admissible initial conditions

$$
x_{t_{0}}=\varphi, \quad \varphi \in C_{+} .
$$

Define a continuous map $f: \mathbb{R}^{1} \times C_{+} \rightarrow R$ by setting

$$
\begin{aligned}
f(t, \varphi) & =-a(t) \varphi(0)+\sum_{j=2}^{m} \beta_{j}(t) \varphi\left(-\tau_{j}(t)\right) e^{-\gamma_{j}(t) \varphi\left(-\tau_{j}(t)\right)} \\
& +\beta_{1}(t) \varphi\left(-\tau_{1}(t)\right) e^{-\gamma_{1}(t) \varphi(0)}-H(t) \varphi(-\sigma(t)) .
\end{aligned}
$$

Then, $f$ is a locally Lipschitz map with respect to $\varphi \in C_{+}$, which ensures the existence and uniqueness of the solution of (1.2) with admissible initial conditions (1.4).

We write $x_{t}\left(t_{0}, \varphi\right)\left(x\left(t ; t_{0}, \varphi\right)\right)$ for an admissible solution of the admissible initial value problem (1.2) and (1.4). Also, let $\left[t_{0}, \eta(\varphi)\right)$ be the maximal right-interval of existence of $x_{t}\left(t_{0}, \varphi\right)$.

Since the function $\frac{1-x}{e^{x}}$ is decreasing with the range $[0,1]$, it follows easily that there exists a unique $\kappa \in(0,1)$ such that

$$
\frac{1-\kappa}{e^{\kappa}}=\frac{1}{e^{2}}
$$

Obviously,

$$
\sup _{x \geq \kappa}\left|\frac{1-x}{e^{x}}\right|=\frac{1}{e^{2}}
$$

Moreover, since $x e^{-x}$ increases on $[0,1]$ and decreases on $[1,+\infty)$, let $\widetilde{\kappa}$ be the unique number in $(1,+\infty)$ such that

$$
\kappa e^{-\kappa}=\widetilde{\kappa} e^{-\widetilde{\kappa}} .
$$

The remaining of this paper is organized as follows. In Section 2, we give some lemmas, which tell us the attracting set for (1.2). These results play an important role in Section 3 to establish the existence of positive periodic solutions of (1.2). Here we also study the global exponential stability of positive periodic solutions. The paper concludes with an example to illustrate the effectiveness of the obtained results by numerical simulation.

\section{Preliminary results}

In this section, we present some lemmas which will play an important role in the next section.

\section{Lemma 2.1 (see [[11], Theorem 2.1]). Assume that}

$$
\inf _{t \in \mathbb{R}}\left\{\beta_{1}(t) e^{-\widetilde{\kappa}}-H(t)\right\}>0, \text { and } \tau_{1}(t) \equiv \sigma(t)
$$

for all $t \in \mathbb{R}$. Then, the solution $x_{t}\left(t_{0}, \varphi\right) \in C_{+}$for all $t \in$ $\left[t_{0}, \eta(\varphi)\right)$, the set of $\left\{x_{t}\left(t_{0}, \varphi\right): t \in\left[t_{0}, \eta(\varphi)\right)\right\}$ is bounded, and $\eta(\varphi)=+\infty$.

Lemma 2.2 (see [[11], Theorem 3.1]) . Suppose that all conditions in Lemma 2.1 are satisfied. Let

$$
\liminf _{t \rightarrow+\infty}\left\{\sum_{j=2}^{m} \frac{\beta_{j}(t)}{a(t)}+\left[\frac{\beta_{1}(t)}{a(t)}-\frac{H(t)}{a(t)}\right]\right\}>1 .
$$

Then, there exist two positive constants $K_{1}$ and $K_{2}$ such that

$$
K_{1} \leq \liminf _{t \rightarrow+\infty} x\left(t ; t_{0}, \varphi\right) \leq \limsup _{t \rightarrow+\infty} x\left(t ; t_{0}, \varphi\right) \leq K_{2} .
$$

Lemma 2.3. Let (2.1) hold. Suppose that there exists a positive constant $M$ such that

$$
\max _{1 \leq j \leq m} \gamma_{j}^{+} \leq \frac{\widetilde{\kappa}}{M}
$$

and

$$
\left\{\begin{array}{l}
\sup _{t \in R}\left\{-a(t)+\frac{1}{e M} \sum_{j=1}^{m} \frac{\beta_{j}(t)}{\gamma_{j}(t)}\right\}<0, \\
\inf _{t \in R}\left\{-a(t)+e^{-\kappa} \sum_{j=2}^{m} \frac{\beta_{j}(t)}{\gamma_{j}(t)}\right\}>0
\end{array} .\right.
$$

Then, the set of $\left\{x_{t}\left(t_{0}, \varphi\right): t \in\left[t_{0}, \eta(\varphi)\right)\right\}$ is bounded, and $\eta(\varphi)=+\infty$. Moreover, there exists $t_{\varphi}>t_{0}$ such that

$$
\kappa<x\left(t ; t_{0}, \varphi\right)<M \text { for all } t \geq t_{\varphi}
$$

Proof. Let $x(t)=x\left(t ; t_{0}, \varphi\right)$. From Lemma 2.1, we have that the set of $\left\{x_{t}\left(t_{0}, \varphi\right): t \in\left[t_{0}, \eta(\varphi)\right)\right\}$ is bounded with $\eta(\varphi)=+\infty$, and

$$
x(t)>0 \text { for all } t \in\left[t_{0},+\infty\right) .
$$


Clearly, (1.3), (2.1) and (2.4) yield to

$$
\begin{aligned}
& 1<e^{\kappa}<\sum_{j=2}^{m} \frac{\beta_{j}(t)}{\gamma_{j}(t) a(t)} \leq \sum_{j=2}^{m} \frac{\beta_{j}(t)}{a(t)} \\
& +\left[\frac{\beta_{1}(t)}{a(t)}-\frac{H(t)}{a(t)}\right] \quad \text { for all } \quad t \in R .
\end{aligned}
$$

It follows from Lemma 2.2 that

$$
0<\liminf _{t \rightarrow+\infty} x(t) \leq \limsup _{t \rightarrow+\infty} x(t)<+\infty
$$

We now prove that $L=\limsup _{t \rightarrow \infty} x(t)<M$. By way of contradiction, we assume that $\stackrel{t \rightarrow \infty}{L} \geq M$. By the fluctuation lemma [[12], Lemma A.1.], there exists a sequence $\left\{\overline{t_{k}}\right\}_{k \geq 1}$ such that

$\overline{t_{k}} \rightarrow+\infty, \quad x\left(\overline{t_{k}}\right) \rightarrow \limsup _{t \rightarrow+\infty} x(t), \quad x^{\prime}\left(\overline{t_{k}}\right) \rightarrow 0 \quad$ as $k \rightarrow+\infty$.

Since $\left\{x_{\overline{t_{k}}}\right\}$ is bounded and equicontinuous, by the AscoliArzelá theorem, there exists a subsequence, still denoted by itself for simplicity of notation, such that

$$
x_{\overline{t_{k}}} \rightarrow \bar{\varphi} \quad \text { for some } \bar{\varphi} \in C_{+} .
$$

Moreover,

$$
\bar{\varphi}(0)=L \geq \bar{\varphi}(\theta) \quad \text { for } \theta \in[-r, 0) .
$$

By the boundedness of the coefficients and delays, there is a subsequence of $\left\{\overline{t_{k}}\right\}$, still denoted by $\left\{\overline{t_{k}}\right\}$, such that $a\left(\overline{t_{k}}\right), \beta_{j}\left(\overline{t_{k}}\right), \tau_{j}\left(\overline{t_{k}}\right)$ and $\gamma_{j}\left(\overline{t_{k}}\right)$ are convergent to $\bar{a}, \bar{\beta}_{j}, \bar{\tau}_{j}$ and $\bar{\gamma}_{j}$, respectively. This can be achieved because of periodicity. In view of $\sup u e^{-u}=\frac{1}{e}$, it follows from

$$
\begin{aligned}
& x^{\prime}\left(\overline{t_{k}}\right) \\
= & -a\left(\overline{t_{k}}\right) x\left(\overline{t_{k}}\right)+\sum_{j=2}^{m} \frac{\beta_{j}\left(\overline{t_{k}}\right)}{\gamma_{j}\left(\overline{t_{k}}\right)} \gamma_{j}\left(\overline{t_{k}}\right) x\left(\overline{t_{k}}-\tau_{j}\left(\overline{t_{k}}\right)\right) e^{-\gamma_{j}\left(\overline{t_{k}}\right) x\left(\overline{t_{k}}-\tau_{j}\left(\overline{t_{k}}\right)\right)} \\
& +\beta_{1}\left(\overline{t_{k}}\right) x\left(\overline{t_{k}}-\tau_{1}\left(\overline{t_{k}}\right)\right) e^{-\gamma_{1}\left(\overline{t_{k}}\right) x\left(\overline{t_{k}}\right)}-H\left(\overline{t_{k}}\right) x\left(\overline{t_{k}}-\sigma\left(\overline{t_{k}}\right)\right) \\
\leq & -a\left(\overline{t_{k}}\right) x\left(\overline{t_{k}}\right)+\sum_{j=2}^{m} \frac{\beta_{j}\left(\overline{t_{k}}\right)}{\overline{\gamma_{j}\left(\overline{t_{k}}\right)}} \gamma_{j}\left(\overline{t_{k}}\right) x\left(\overline{t_{k}}-\tau_{j}\left(\overline{t_{k}}\right)\right) e^{-\gamma_{j}\left(\overline{t_{k}}\right) x\left(\overline{t_{k}}-\tau_{j}\left(\overline{t_{k}}\right)\right)} \\
& +\beta_{1}\left(\overline{t_{k}}\right) x\left(\overline{t_{k}}-\tau_{1}\left(\overline{t_{k}}\right)\right) e^{-\gamma_{1}\left(\overline{t_{k}}\right) x\left(\overline{t_{k}}\right)}
\end{aligned}
$$

that (taking limits)

$$
\begin{aligned}
0 & \leq-\bar{a} L+\sum_{j=2}^{m} \frac{\bar{\beta}_{j}}{\bar{\gamma}_{j}} \overline{\gamma_{j}} \bar{\varphi}\left(-\bar{\tau}_{j}\right) e^{-\overline{\gamma_{j}} \bar{\varphi}\left(-\bar{\tau}_{j}\right)}+\frac{\overline{\beta_{1}}}{\bar{\gamma}_{1}} \overline{\gamma_{1}} \bar{\varphi}\left(-\bar{\tau}_{1}\right) e^{-\bar{\gamma}_{1} L} \\
& \leq-\bar{a} L+\sum_{j=2}^{m} \frac{\overline{\beta_{j}}}{\bar{\gamma}_{j}} \overline{\gamma_{j}} \bar{\varphi}\left(-\bar{\tau}_{j}\right) e^{-\overline{\gamma_{j}} \bar{\varphi}\left(-\bar{\tau}_{j}\right)}+\frac{\overline{\beta_{1}}}{\bar{\gamma}_{1}} \overline{\gamma_{1}} L e^{-\bar{\gamma}_{1} L} \\
& \leq-\bar{a} M+\sum_{j=1}^{m} \frac{\bar{\beta}_{j}}{\bar{\gamma}_{j}} \frac{1}{e} \\
& \leq M \sup \left\{-a(t)+\frac{1}{e M} \sum_{j=1}^{m} \frac{\beta_{j}(t)}{\gamma_{j}(t)}\right\} \\
& <0
\end{aligned}
$$

a contradiction and hence $L=\limsup _{t \rightarrow \infty} x(t)<M$. Then, we can choose $t^{\#}>t_{0}$ such that

$$
x(t)<M, \text { for all } t \geq t^{\#} .
$$

We finally show that $l=\liminf x(t)>\kappa$. By way of contradiction, we assume that $0<l \leq \kappa$. Again from the fluctuation lemma [12, Lemma A.1.], there exists a sequence $\left\{t_{k}\right\}_{k \geq 1}$ such that

$$
t_{k} \geq t^{\#}, k=1,2, \cdots \cdots
$$

and

$t_{k} \rightarrow+\infty, \quad x\left(t_{k}\right) \rightarrow \liminf _{t \rightarrow+\infty} x(t), \quad x^{\prime}\left(t_{k}\right) \rightarrow 0 \quad$ as $k \rightarrow+\infty$.

Since $\left\{x_{t_{k}}\right\}$ is bounded and equicontinuous, by the AscoliArzelá theorem, there exists a subsequence, still denoted by itself for simplicity of notation, such that

$$
x_{t_{k}} \rightarrow \varphi^{*} \quad \text { for some } \varphi^{*} \in C_{+} .
$$

Moreover,

$$
\varphi^{*}(0)=l \leq \varphi^{*}(\theta) \leq M \quad \text { for } \theta \in[-r, 0)
$$

Without loss of generality, we assume that all $a\left(t_{k}\right), \beta_{j}\left(t_{k}\right)$, $\tau_{j}\left(t_{k}\right)$ and $\gamma_{j}\left(t_{k}\right)$ are convergent to $a^{* *}, \beta_{j}^{* *}, \tau_{j}^{* *}$ and $\gamma_{j}^{* *}$, respectively. This can be achieved because of periodicity. Then, (1.3), (2.3) and (2.9) lead to

$$
\gamma_{1}\left(t_{k}\right) x\left(t_{k}\right) \leq \gamma_{1}^{+} M \leq \widetilde{\kappa}, k=1,2, \cdots
$$

and

$$
l \leq \gamma_{j}^{*} \varphi^{*}\left(-\tau_{j}^{*}\right) \leq \gamma_{j}^{*} M \leq \widetilde{\kappa}, \quad j=2,3, \cdots, m .
$$

It follows from

$$
\begin{aligned}
x^{\prime}\left(t_{k}\right)= & -a\left(t_{k}\right) x\left(t_{k}\right) \\
& +\sum_{j=2}^{m} \frac{\beta_{j}\left(t_{k}\right)}{\gamma_{j}\left(t_{k}\right)} \gamma_{j}\left(t_{k}\right) x\left(t_{k}-\tau_{j}\left(t_{k}\right)\right) e^{-\gamma_{j}\left(t_{k}\right) x\left(t_{k}-\tau_{j}\left(t_{k}\right)\right)} \\
& +x\left(t_{k}-\tau_{1}\left(t_{k}\right)\right)\left[\beta_{1}\left(t_{k}\right) e^{-\gamma_{1}\left(t_{k}\right) x\left(t_{k}\right)}-H\left(t_{k}\right)\right] \\
\geq & -a\left(t_{k}\right) x\left(t_{k}\right) \\
& +\sum_{j=2}^{m} \frac{\beta_{j}\left(t_{k}\right)}{\gamma_{j}\left(t_{k}\right)} \gamma_{j}\left(t_{k}\right) x\left(t_{k}-\tau_{j}\left(t_{k}\right)\right) e^{-\gamma_{j}\left(t_{k}\right) x\left(t_{k}-\tau_{j}\left(t_{k}\right)\right)} \\
& +x\left(t_{k}-\tau_{1}\left(t_{k}\right)\right)\left[\beta_{1}\left(t_{k}\right) e^{-\widetilde{\kappa}}-H\left(t_{k}\right)\right] \\
\geq & -a\left(t_{k}\right) x\left(t_{k}\right) \\
& +\sum_{j=2}^{m} \frac{\beta_{j}\left(t_{k}\right)}{\gamma_{j}\left(t_{k}\right)} \gamma_{j}\left(t_{k}\right) x\left(t_{k}-\tau_{j}\left(t_{k}\right)\right) e^{-\gamma_{j}\left(t_{k}\right) x\left(t_{k}-\tau_{j}\left(t_{k}\right)\right)}
\end{aligned}
$$


that (taking limits)

$$
\begin{aligned}
0 & \geq-a^{* *} l+\sum_{j=2}^{m} \frac{\beta_{j}^{* *}}{\gamma_{j}^{* *}} \gamma_{j}^{* *} \varphi^{*}\left(-\tau_{j}^{* *}\right) e^{-\gamma_{j}^{* *} \varphi^{*}\left(-\tau_{j}^{* *}\right)} \\
& \geq-a^{* *} l+\sum_{j=2}^{m} \frac{\beta_{j}^{* *}}{\gamma_{j}^{* *}} l e^{-l} \\
& \geq \operatorname{linf}_{t \in R}\left\{-a(t)+\sum_{j=2}^{m} \frac{\beta_{j}(t)}{\gamma_{j}(t)} e^{-l}\right\} \\
& \geq \operatorname{linf}_{t \in R}\left\{-a(t)+\sum_{j=2}^{m} \frac{\beta_{j}(t)}{\gamma_{j}(t)} e^{-\kappa}\right\} \\
& >0
\end{aligned}
$$

a contradiction. This proves that $l>\kappa$. Hence, from (2.9), we can choose $t_{\varphi}>t_{0}$ such that

$$
\kappa<x\left(t ; t_{0}, \varphi\right)<M \text { for all } t \geq t_{\varphi}
$$

This ends the proof of Lemma 2.3.

Lemma 2.4. Suppose (2.1), (2.3) and (2.4) hold, and

$\sup _{t \in R}\left\{-a(t)+\sum_{j=2}^{m} \beta_{j}(t) \frac{1}{e^{2}}+\beta_{1}(t) e^{-\kappa}(M+1)+H(t)\right\}<0$.

Moreover, let $x^{*}(t)=x\left(t ; t_{0}, \varphi^{*}\right), \quad x(t)=x\left(t ; t_{0}, \varphi\right)$. Then, there exists a positive constant $\lambda$ such that

$$
x(t)-x^{*}(t)=O\left(e^{-\lambda t}\right) .
$$

Proof. Define a continuous function $\Gamma$ by setting

$$
\begin{aligned}
& \Gamma(\mu)=\sup _{t \in R}\left\{-[a(t)-\mu]+\sum_{j=2}^{m} \beta_{j}(t) \frac{1}{e^{2}} e^{\mu r}\right. \\
& \left.+\beta_{1}(t) e^{-\kappa}\left(M+e^{\mu r}\right)+H(t) e^{\mu r}\right\}, \mu \in[0,1] .
\end{aligned}
$$

Then, we have

$$
\begin{aligned}
& \Gamma(0)=\sup _{t \in R}\left\{-a(t)+\sum_{j=2}^{m} \beta_{j}(t) \frac{1}{e^{2}}\right. \\
& \left.+\beta_{1}(t) e^{-\kappa}(M+1)+H(t)\right\}<0
\end{aligned}
$$

which implies that there exist two constants $\eta>0$ and $\lambda \in$ $(0,1]$ such that

$$
\begin{gathered}
\Gamma(\lambda)=\sup _{t \in R}\left\{-[a(t)-\lambda]+\sum_{j=2}^{m} \beta_{j}(t) \frac{1}{e^{2}} e^{\lambda r}\right. \\
\left.+\beta_{1}(t) e^{-\kappa}\left(M+e^{\lambda r}\right)+H(t) e^{\lambda r}\right\}<-\eta<0 .
\end{gathered}
$$

Set $y(t)=x(t)-x^{*}(t)$, where $t \in\left[t_{0}-r,+\infty\right)$. Then

$$
\begin{aligned}
y^{\prime}(t)= & -a(t) y(t) \\
& +\sum_{j=2}^{m} \beta_{j}(t)\left[x\left(t-\tau_{j}(t)\right) e^{-\gamma_{j}(t) x\left(t-\tau_{j}(t)\right)}\right. \\
& \left.-x^{*}\left(t-\tau_{j}(t)\right) e^{-\gamma_{j}(t) x^{*}\left(t-\tau_{j}(t)\right)}\right] \\
& +\beta_{1}(t)\left[x\left(t-\tau_{1}(t)\right) e^{-\gamma_{1}(t) x(t)}\right. \\
& \left.-x^{*}\left(t-\tau_{1}(t)\right) e^{-\gamma_{1}(t) x^{*}(t)}\right]-H(t) y(t-\sigma(t)) .
\end{aligned}
$$

It follows from Lemma 2.3 that there exists $t_{\varphi, \varphi^{*}}>t_{0}$ such that

$$
\kappa \leq x(t), x^{*}(t) \leq M, \text { for all } t \in\left[t_{\varphi, \varphi^{*}}-r,+\infty\right) .
$$

We consider the Lyapunov functional

$$
V(t)=|y(t)| e^{\lambda t} .
$$

Calculating the upper left derivative of $V(t)$ along the solution $y(t)$ of (2.13), we have

$$
\begin{aligned}
& D^{-}(V(t)) \\
\leq & -a(t)|y(t)| e^{\lambda t} \\
& +\sum_{j=2}^{m} \beta_{j}(t) \mid x\left(t-\tau_{j}(t)\right) e^{-\gamma_{j}(t) x\left(t-\tau_{j}(t)\right)} \\
& -x^{*}\left(t-\tau_{j}(t)\right) e^{-\gamma_{j}(t) x^{*}\left(t-\tau_{j}(t)\right)} \mid e^{\lambda t} \\
& +\beta_{1}(t)\left|x\left(t-\tau_{1}(t)\right) e^{-\gamma_{1}(t) x(t)}-x^{*}\left(t-\tau_{1}(t)\right) e^{-\gamma_{1}(t) x^{*}(t)}\right| e^{\lambda t} \\
& +H(t)|y(t-\sigma(t))| e^{\lambda t}+\lambda|y(t)| e^{\lambda t}, \text { for all } t>t_{\varphi, \varphi^{*}} \cdot(2.16)
\end{aligned}
$$

We claim that

$$
\begin{aligned}
V(t) & =|y(t)| e^{\lambda t} \\
& <e^{\lambda t_{\varphi, \varphi^{*}}}\left(\max _{t \in\left[t_{0}-r, t_{\varphi, \varphi^{*}}\right]}\left|x(t)-x^{*}(t)\right|+1\right) \\
& :=K_{\varphi, \varphi^{*}} \text { for all } t>t_{\varphi, \varphi^{*}} .(2.17)
\end{aligned}
$$

Contrarily, there must exist $t_{*}>t_{\varphi, \varphi^{*}}$ such that

$V\left(t_{*}\right)=K_{\varphi, \varphi^{*}}$ and $V(t)<K_{\varphi, \varphi^{*}}$ for all $t \in\left[t_{0}-r, t_{*}\right)$.

Since

$$
\begin{gathered}
\kappa \leq \gamma_{j}\left(t_{*}\right) x\left(t_{*}-\tau_{j}\left(t_{*}\right)\right), \gamma_{j}\left(t_{*}\right) x^{*}\left(t_{*}-\tau_{j}\left(t_{*}\right)\right) \\
\leq \gamma_{j}^{+} M \leq \widetilde{\kappa}, \quad j=1,2, \cdots, m
\end{gathered}
$$

Together with (1.5), (1.6), (1.7), (2.16), (2.18) and the inequalities

$$
\begin{aligned}
\left|e^{-s}-e^{-t}\right| & =e^{-(s+\theta(t-s))}|s-t| \\
& \leq e^{-\kappa}|s-t| \text { where } s, t \in[\kappa, \widetilde{\kappa}], 0<\theta<1
\end{aligned}
$$

and

$\left|s e^{-s}-t e^{-t}\right|=\left|\frac{1-(s+\theta(t-s))}{e^{s+\theta(t-s)}}\right||s-t|$ 


$$
\leq \frac{1}{e^{2}}|s-t| \text { where } s, t \in[\kappa,+\infty), 0<\theta<1,
$$

we obtain

$$
\begin{aligned}
& 0 \leq D^{-}\left(V\left(t_{*}\right)\right) \\
& \leq-a\left(t_{*}\right)\left|y\left(t_{*}\right)\right| e^{\lambda t_{*}} \\
& +\sum_{j=2}^{m} \beta_{j}\left(t_{*}\right) \mid x\left(t_{*}-\tau_{j}\left(t_{*}\right)\right) e^{-\gamma_{j}\left(t_{*}\right) x\left(t_{*}-\tau_{j}\left(t_{*}\right)\right)} \\
& -x^{*}\left(t_{*}-\tau_{j}\left(t_{*}\right)\right) e^{-\gamma_{j}\left(t_{*}\right) x^{*}\left(t_{*}-\tau_{j}\left(t_{*}\right)\right)} \mid e^{\lambda t_{*}} \\
& +\beta_{1}\left(t_{*}\right) \mid x\left(t_{*}-\tau_{1}\left(t_{*}\right)\right) e^{-\gamma_{1}\left(t_{*}\right) x\left(t_{*}\right)} \\
& -x^{*}\left(t_{*}-\tau_{1}\left(t_{*}\right)\right) e^{-\gamma_{1}\left(t_{*}\right) x^{*}\left(t_{*}\right)} \mid e^{\lambda t_{*}} \\
& +H\left(t_{*}\right)\left|y\left(t_{*}-\sigma\left(t_{*}\right)\right)\right| e^{\lambda t_{*}}+\lambda\left|y\left(t_{*}\right)\right| e^{\lambda t_{*}} \\
& \leq-a\left(t_{*}\right)\left|y\left(t_{*}\right)\right| e^{\lambda t_{*}} \\
& +\sum_{j=2}^{m} \frac{\beta_{j}\left(t_{*}\right)}{\gamma_{j}\left(t_{*}\right)} \mid \gamma_{j}\left(t_{*}\right) x\left(t_{*}-\tau_{j}\left(t_{*}\right)\right) e^{-\gamma_{j}\left(t_{*}\right) x\left(t_{*}-\tau_{j}\left(t_{*}\right)\right)} \\
& -\gamma_{j}\left(t_{*}\right) x^{*}\left(t_{*}-\tau_{j}\left(t_{*}\right)\right) e^{-\gamma_{j}\left(t_{*}\right) x^{*}\left(t_{*}-\tau_{j}\left(t_{*}\right)\right)} \mid e^{\lambda t_{*}} \\
& +\beta_{1}\left(t_{*}\right) \mid x\left(t_{*}-\tau_{1}\left(t_{*}\right)\right) e^{-\gamma_{1}\left(t_{*}\right) x\left(t_{*}\right)} \\
& -x\left(t_{*}-\tau_{1}\left(t_{*}\right)\right) e^{-\gamma_{1}\left(t_{*}\right) x^{*}\left(t_{*}\right)} \mid e^{\lambda t_{*}} \\
& +\beta_{1}\left(t_{*}\right) \mid x\left(t_{*}-\tau_{1}\left(t_{*}\right)\right) e^{-\gamma_{1}\left(t_{*}\right) x^{*}\left(t_{*}\right)} \\
& -x^{*}\left(t_{*}-\tau_{1}\left(t_{*}\right)\right) e^{-\gamma_{1}\left(t_{*}\right) x^{*}\left(t_{*}\right)} \mid e^{\lambda t_{*}} \\
& +H\left(t_{*}\right)\left|y\left(t_{*}-\sigma\left(t_{*}\right)\right)\right| e^{\lambda t_{*}}+\lambda\left|y\left(t_{*}\right)\right| e^{\lambda t_{*}} \\
& \leq-\left[a\left(t_{*}\right)-\lambda\right]\left|y\left(t_{*}\right)\right| e^{\lambda t_{*}}+\sum_{j=2}^{m} \beta_{j}\left(t_{*}\right) \frac{1}{e^{2}}\left|y\left(t_{*}-\tau_{j}\left(t_{*}\right)\right)\right| e^{\lambda t_{*}} \\
& +\beta_{1}\left(t_{*}\right) M\left|e^{-\gamma_{1}\left(t_{*}\right) x\left(t_{*}\right)}-e^{-\gamma_{1}\left(t_{*}\right) x^{*}\left(t_{*}\right)}\right| e^{\lambda t_{*}} \\
& +\beta_{1}\left(t_{*}\right)\left|x\left(t_{*}-\tau_{1}\left(t_{*}\right)\right)-x^{*}\left(t_{*}-\tau_{1}\left(t_{*}\right)\right)\right| e^{-\gamma_{1}\left(t_{*}\right) x^{*}\left(t_{*}\right)} e^{\lambda t_{*}} \\
& +H\left(t_{*}\right)\left|y\left(t_{*}-\sigma\left(t_{*}\right)\right)\right| e^{\lambda t_{*}} \\
& \leq-\left[a\left(t_{*}\right)-\lambda\right]\left|y\left(t_{*}\right)\right| e^{\lambda t_{*}} \\
& +\sum_{j=2}^{m} \beta_{j}\left(t_{*}\right) \frac{1}{e^{2}}\left|y\left(t_{*}-\tau_{j}\left(t_{*}\right)\right)\right| e^{\lambda\left(t_{*}-\tau_{j}\left(t_{*}\right)\right)} e^{\lambda \tau_{j}\left(t_{*}\right)} \\
& +\beta_{1}\left(t_{*}\right) M e^{-\kappa}\left|y\left(t_{*}\right)\right| e^{\lambda t_{*}} \\
& +\beta_{1}\left(t_{*}\right) e^{-\kappa}\left|y\left(t_{*}-\tau_{1}\left(t_{*}\right)\right)\right| e^{\lambda\left(t_{*}-\tau_{1}\left(t_{*}\right)\right)} e^{\lambda \tau_{1}\left(t_{*}\right)} \\
& +H\left(t_{*}\right)\left|y\left(t_{*}-\sigma\left(t_{*}\right)\right)\right| e^{\lambda\left(t_{*}-\sigma\left(t_{*}\right)\right)} e^{\lambda \sigma\left(t_{*}\right)} \\
& \leq\left\{-\left[a\left(t_{*}\right)-\lambda\right]+\sum_{j=2}^{m} \beta_{j}\left(t_{*}\right) \frac{1}{e^{2}} e^{\lambda r}\right. \\
& \left.+\beta_{1}\left(t_{*}\right) e^{-\kappa}\left(M+e^{\lambda r}\right)+H\left(t_{*}\right) e^{\lambda r}\right\} K_{\varphi, \varphi^{*}} .
\end{aligned}
$$

Thus,

$$
\begin{aligned}
& 0 \leq-\left[a\left(t_{*}\right)-\lambda\right]+\sum_{j=2}^{m} \beta_{j}\left(t_{*}\right) \frac{1}{e^{2}} e^{\lambda r} \\
& +\beta_{1}\left(t_{*}\right) e^{-\kappa}\left(M+e^{\lambda r}\right)+H\left(t_{*}\right) e^{\lambda r}
\end{aligned}
$$

which contradicts with (2.12). Hence, (2.17) holds. It follows that

$$
|y(t)|<K_{\varphi, \varphi^{*}} e^{-\lambda t} \text { for all } t>t_{\varphi, \varphi^{*}}
$$

This completes the proof of Lemma 2.4.

\section{Main Results}

In this section, we establish sufficient conditions on the existence, uniqueness, and global exponential stability of positive $T$-periodic solutions of (1.2).

Theorem 3.1. Suppose that all conditions in Lemma 2.4 are satisfied. Then (1.2) has exactly one $T$ - periodic solution $x^{*}(t)$. Moreover, $x^{*}(t)$ is globally exponentially stable, i. e., there exist constants $\lambda>0, K>0$ and $T^{*}>t_{0}$ such that

$$
\left|x\left(t ; t_{0}, \varphi\right)-x^{*}(t)\right|<K e^{-\lambda t} \text { for all } t>T^{*} .
$$

Proof. Let $x(t)=x\left(t ; t_{0}, \varphi\right)$. By Lemma 2.3, we obtain that there exists $T_{0}>t_{0}$ such that

$$
\kappa<x(t)<M, \text { for all } t \geq T_{0}-r \text {. }
$$

By the periodicity of coefficients and delays for (1.2), we have, for any natural number $h$

$$
\begin{aligned}
& {[x(t+(h+1) T)]^{\prime} } \\
= & -a(t+(h+1) T) x(t+(h+1) T) \\
& +\sum_{j=2}^{m} \beta_{j}(t+(h+1) T) x\left(t+(h+1) T-\tau_{j}(t+(h+1) T)\right) \\
& \times e^{-\gamma_{j}(t+(h+1) T) x\left(t+(h+1) T-\tau_{j}(t+(h+1) T)\right)} \\
& +\beta_{1}(t+(h+1) T) x\left(t+(h+1) T-\tau_{1}(t+(h+1) T)\right) \\
& \times e^{-\gamma_{1}(t+(h+1) T) x(t+(h+1) T)} \\
& -H(t+(h+1) T) x(t+(h+1) T-\sigma(t+(h+1) T)) \\
= & -a(t) x(t+(h+1) T) \\
& +\sum_{j=2}^{m} \beta_{j}(t) x\left(t+(h+1) T-\tau_{j}(t)\right) e^{-\gamma_{j}(t) x\left(t+(h+1) T-\tau_{j}(t)\right)} \\
& +\beta_{1}(t) x\left(t+(h+1) T-\tau_{1}(t)\right) e^{-\gamma_{1}(t) x(t+(h+1) T)} \\
& -H(t) x(t+(h+1) T-\sigma(t)), \quad t+(h+1) T \in\left[t_{0},+\infty\right) .
\end{aligned}
$$

Thus, for any natural number $h$, we obtain that $x(t+(h+$ 1)T) is a solution of (1.2) for all $t \geq t_{0}-r-(h+1) T$. Hence, $x(t+T)\left(t \in\left[t_{0}-r,+\infty\right)\right)$ is also a solution of (1.2) with initial values

$$
\psi(s)=x\left(s+t_{0}+T\right), s \in[-r, 0] .
$$

It follows from the proof of Lemma 2.4 that, for any nonnegative integer $h$ and $t+h T \geq T_{0}$,

$$
\begin{aligned}
& \left|x\left(t+(h+1) T ; t_{0}, \varphi\right)-x\left(t+h T ; t_{0}, \varphi\right)\right| \\
= & \left|x\left(t+h T ; t_{0}, \psi\right)-x\left(t+h T ; t_{0}, \varphi\right)\right| \\
\leq & K_{\varphi, \psi} e^{-\lambda(t+h T)}
\end{aligned}
$$

where

$K_{\varphi, \psi}=e^{\lambda T_{0}}\left(\max _{s \in\left[t_{0}-r, T_{0}\right]}\left|x\left(s ; t_{0}, \psi\right)-x\left(s ; t_{0}, \varphi\right)\right|+1\right)$.

Now, we show that $x\left(t+q T ; t_{0}, \varphi\right)$ is convergent on any compact interval as $q \rightarrow+\infty$. Let $[a, b] \subset R$ be an arbitrary subset of $R$. Choose a nonnegative integer $q_{0}$ such 
that $t+q_{0} T \geq T_{0}$ for $t \in[a, b]$. Then for $t \in[a, b]$ and $q>q_{0}$ we have

$x(t+q T)=x\left(t+q_{0} T\right)+\sum_{h=q_{0}}^{q-1}[x(t+(h+1) T)-x(t+h T)]$.

Then $x(t+q T)$ will converge uniformly to a continuous function, say $x^{*}(t)$, on $[a, b]$. Because of arbitrariness of $[a, b]$, we see that $x(t+q T) \rightarrow x^{*}(t)$ as $q \rightarrow+\infty$ for $t \in R$. Moreover,

$$
\kappa \leq x^{*}(t) \leq M \quad \text { for all } t \in R .
$$

It remains to show that $x^{*}$ is a $T$-periodic solution of (1.2). The periodicity is obvious since

$$
\begin{aligned}
& x^{*}(t+T)=\lim _{q \rightarrow+\infty} x((t+T)+q T) \\
& =\lim _{q+1 \rightarrow+\infty} x(t+(q+1) T)=x^{*}(t)
\end{aligned}
$$

for all $t \in R$. Now, note that $x(t+q T)$ is a solution to (1.2), that is,

$$
\begin{aligned}
& x(t+q T)-x\left(t_{0}+q T\right) \\
& =\int_{t_{0}}^{t}[-a(s) x(s+q T) \\
& +\sum_{j=2}^{m} \beta_{j}(s) x\left(s+q T-\tau_{j}(s)\right) e^{-\gamma_{j}(s) x\left(s+q T-\tau_{j}(s)\right)} \\
& +\beta_{1}(s) x\left(s+q T-\tau_{1}(s)\right) e^{-\gamma_{1}(s) x(s+q T)} \\
& -H(s) x(s+q T-\sigma(s))] d s
\end{aligned}
$$

for $t \geq t_{0}$. Letting $q \rightarrow+\infty$ gives us

$$
\begin{gathered}
x^{*}(t)-x^{*}\left(t_{0}\right) \\
=\int_{t_{0}}^{t}\left[-a(s) x^{*}(s)+\sum_{j=2}^{m} \beta_{j}(s) x^{*}\left(s-\tau_{j}(s)\right) e^{-\gamma_{j}(s) x^{*}\left(s-\tau_{j}(s)\right)}\right. \\
\left.+\beta_{1}(s) x^{*}\left(s-\tau_{1}(s)\right) e^{-\gamma_{1}(s) x^{*}(s)}-H(s) x^{*}(s-\sigma(s))\right] d s
\end{gathered}
$$

for $t \geq t_{0}$, namely, $x^{*}$ is a solution to (1.2) on $\left[t_{0}-r,+\infty\right)$.

Finally, from (3.3), again using a similar argument as in (2.28) of Lemma 2.3, we can prove that (3.1) holds. This completes the proof.

\section{An example}

In this section, we present an example to check the validity of our results we obtained in the previous sections.

Example 4.1. Consider the following Nicholson's blowflies model with a linear harvesting term:

$$
\begin{gathered}
x^{\prime}(t)=-\frac{30+15|\cos t|}{100} x(t) \\
+\frac{100-\sin t}{100+\sin t} x\left(t-2 e^{\sin ^{4} t}\right) e^{-x\left(t-2 e^{\sin ^{4} t}\right)}
\end{gathered}
$$

$$
\begin{aligned}
+ & \frac{1}{100}\left(3+\cos ^{4} t\right) x\left(t-2 e^{\cos ^{4} t}\right) e^{-x(t)} \\
& -\frac{1}{100}\left(2+\cos ^{4} t\right) x\left(t-2 e^{\cos ^{4} t}\right) .
\end{aligned}
$$

Obviously,

$$
\begin{gathered}
a^{+}=0.45, a^{-}=0.3, \quad \beta_{2}^{-}=\frac{99}{101}, \quad \beta_{2}^{+}=\frac{101}{99}, \\
\gamma_{i}^{-}=\gamma_{i}^{+}=1, i=1,2, \\
\beta_{1}(t)=\frac{1}{100}\left(3+\cos ^{4} t\right), H(t)=\frac{1}{100}\left(2+\cos ^{4} t\right) \\
\tau_{2}(t)=2 e^{\sin ^{4} t}, \quad \tau_{1}(t)=\sigma(t)=2 e^{\cos ^{4} t}, \quad r=2 e .
\end{gathered}
$$

Note $\kappa \approx 0.7215355$ and $\tilde{\kappa} \approx 1.342276$. Let $M=1.33$. Then

$$
\begin{aligned}
& a^{-} M=0.3 \times 1.33 \approx 0.399 \\
& \frac{\beta_{2}^{+}}{\gamma_{2}^{-}} \frac{1}{e}=\frac{101}{99} \frac{1}{e} \approx 0.3753113, \frac{\beta_{1}^{+}}{\gamma_{1}^{-}} \frac{1}{e}=\frac{101}{99} \frac{1}{e} \approx 0.016 \\
& \frac{\beta_{2}^{-}}{\gamma_{2}^{+}} e^{-\kappa}=\frac{99}{101} e^{-\kappa} \approx \frac{99}{101} e^{-0.7215355} \approx 0.4763816 \\
& \beta_{2}^{+} \frac{1}{e^{2}}=\frac{101}{99} \frac{1}{e^{2}} \approx 0.1380693 \\
& \beta_{1}^{+} e^{-\kappa}(M+1)+H^{+} \approx 0.09
\end{aligned}
$$

which imply that (4.1) satisfies the assumptions of Theorem 3.1. Therefore, equation (4.1) has a unique positive $2 \pi$-periodic solution $x^{*}(t)$, which is globally exponentially stable with the exponential convergent rate $\lambda \approx 0.005$. The numerical simulation in Fig. 1 strongly supports the conclusion.

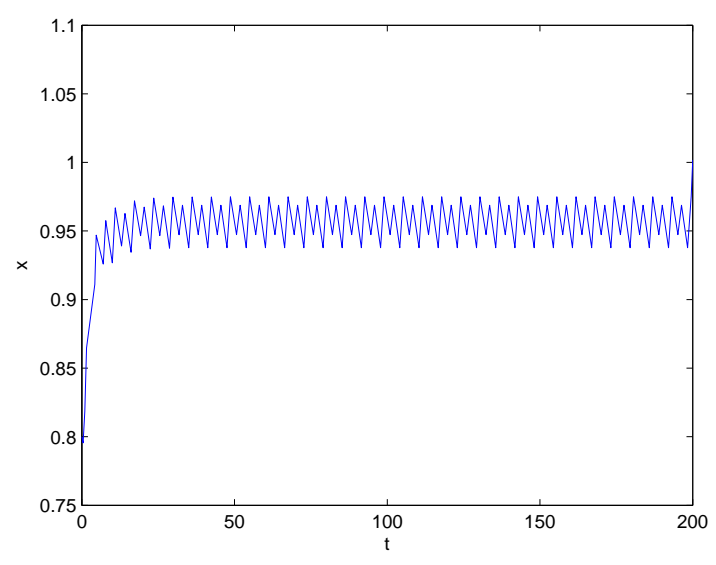

Fig. 1: Numerical solution $x(t)$ of equation (4.1) for initial value $\varphi(s) \equiv 0.8, s \in[-2 e, 0]$.

Remark 4.1. To the best of our knowledge, few authors have considered the problems on the global 
dynamic behaviors of positive periodic solution for Nicholson's blowflies model with a linear harvesting term. It is clear that all the results in $[4-10,13]$ and the references therein cannot be applicable to prove the global stability of positive $2 \pi$-periodic solution for (4.1). Moreover, in this present paper, we employ a novel proof to establish some criteria to guarantee the existence and global exponential stability of positive periodic solutions for Nicholsons blowflies model with a linear harvesting term.

\section{Acknowledgement}

This work was supported by the Scientific Research Fund of Hunan Provincial Natural Science Foundation of PR China (Grant No. 11JJ6006), the Natural Scientific Research Fund of Hunan Provincial Education Department of PR China (Grants No. 11C0916, 11C0915).

The authors are grateful to the anonymous referee for a careful checking of the details and for helpful comments that improved this paper.

\section{References}

[1] W. Gurney, S. Blythe, R. Nisbet, Nicholsons blowflies revisited, Nature, 287, 17-21 (1980).

[2] A. Nicholson, An outline of the dynamics of animal populations, Aust. J. Zool., 2, 9-65 (1954).

[3] L. Berezansky, E. Braverman, L. Idels, Nicholson's Blowflies Differential Equations Revisited: Main Results and Open Problems, Appl. Math. Modelling, 34, 1405-1417 (2010).

[4] F. Long, M. Yang, Positive periodic solutions of delayed Nicholson's blowflies model with a linear harvesting term, Electron. J. Qual. Theory Differ. Equ., 41, 1-11 (2011).

[5] P. Amster, A. Déboli, Existence of positive -periodic solutions of a generalized Nicholsons blowflies model with a nonlinear harvesting term , Appl. Math. Lett., 25, 1203-1207 (2012).

[6] W. Zhao, C. Zhu, H. Zhu, On positive periodic solution for the delay Nicholsons blowflies model with a harvesting term Appl. Math. Modell., 36, 3335-3340 (2012).

[7] Q. Zhou, The positive periodic solution for Nicholson-type delay system with linear harvesting terms, Appl. Math. Modell., 37, 5581-5590 (2013).

[8] X. Liu, J. Meng, The Positive Almost Periodic Solution for Nicholson-type Delay Systems with Linear Harvesting Terms, Appl. Math. Modell., 36, 3289-3298 (2012).

[9] F. Long, Positive almost periodic solution for a class of Nicholsons blowflies model with a linear harvesting term, Nonlinear Anal. Real World Appl., 13, 686-693 (2012).

[10] L. Wang, Almost periodic solution for Nicholson's blowflies model with patch structure and linear harvesting terms, Appl. Math. Modell., 37, 2153-2165 (2013).

[11] B. Liu, Global dynamic behaviors for a delayed Nicholson's blowflies model with a linear harvesting term, Electron. J. Qual. Theory Differ. Equ., 45, 1-13 (2013).
[12] H. L. Smith, An Introduction to Delay Differential Equations with Applications to the Life Sciences, Springer New York, (2011).

[13] B. Liu, Global exponential stability of positive periodic solutions for a delayed Nicholson's blowflies model, J. Math. Anal. Appl., 412, 212-221 (2014).

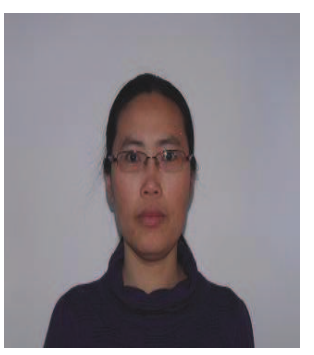

Ani Jiang received the B.S. degree in mathematics, from Tianjin normal University, Tianjin China, in 2000, and the M.S. degrees in applied mathematics from Hunan University, Changsha China, in 2006, respectively. She is currently an Associate Professor in the College of Mathematics and Computer Science, Hunan University of Arts and Science, Changde, Hunan 415000, P. R. China. She is also the author or collaborator of more than 2 journal papers. Her research interests are in the areas of dynamics of neural networks, and qualitative theory of differential equations and difference equations. 